\title{
Bioinformatics Designing of 10-23 Deoxyribozyme against Coding Region of Beta-galactosidase Gene
}

\author{
Nasrin al-Sadat Ahmadi, Abolghasem Esmaeili *, Fatemeh Javadi-Zarnaghi \\ Cellular and Molecular Biology Division; Department of Biology; Faculty of Sciences, University of Isfahan, Isfahan, Iran.
}

Received: 25 Jan 2016

Revised : 18 Feb 2016

Accepted: 27 Feb 2016

Corresponding Author: Abolghasem Esmaeili

Cellular and Molecular Biology Division; Department of Biology; Faculty of Sciences, University of Isfahan, Isfahan, Iran.

Phone: +98-3137932490

E-mail: aesmaeili@sci.ui.ac.ir

\begin{abstract}
Background: Deoxyribozymes (Dzs) can play a role as gene expression inhibitors at mRNA level. Among Dzs, the 10-23 deoxyribozyme has significant potentials for treatment of diseases. Designed Dz includes a catalytic core made of 15 deoxyribonucleotides and two binding arms consisted of 6-12 nucleotides for site specific binding to target RNA and hydrolysis. The enzyme has characteristic features for cleavage of the RNA target between an unpaired purine (A, G) and a paired pyrimidine ( $\mathrm{C}$ or $\mathrm{U}$ ). In this study, $10-23 \mathrm{Dz}$ is designed for the coding region of the $\alpha$-peptide of a lac $Z$ gene.

Materials and Methods: The primary sequence of a plasmid with $\alpha$ complementation ability was taken from addgene database. To confirm sequence validity, ExPASy was used to analyze related ORFs for the retrieved sequence. The ORF with identical sequence to $\alpha$-peptide was selected in the reverse complement sequence. Subsequently, the secondary structure of the $\alpha$-peptide was analyzed in DINAMelt web server and Mfold software. Then the intended target site was selected inside the coding region of the $\alpha$-peptide. The Dzs sequence was designed for the target site with nucleotide binding arms.

Results and conclusion: The resulted Dz in this study can be used as a promising catalytic DNA inside bacterial cells for blue-white screening. Criteria such as biological stability and catalytic rate of such enzymes must be evaluated in vivo and in vitro.
\end{abstract}

Keywords: 10-23 deoxyribozyme; Gene expression; beta-galactosidase gene

Please cite this article as: Ahmadi N, Esmaeili A, Javadi Zarnaghi F. Bioinformatics Designing of 10-23 Deoxyribozyme against Coding Region of Beta-galactosidase Gene. Res Mol Med. 2016; 5 (2): 28-33

\section{Introduction}

Deoxyribozymes (Dzs) are widely acknowledged as promising oligonucleotides for selective inhibition of gene expression at mRNA level (1). Among deoxyribozymes, Dz 10-23 and Dz 8-17 have more often been applied in research fields. The Dz 10-23 is investigated in this study. The "10-23" contains 15 nucleotides in the catalytic core, flanked by complementary binding arms of 6-12 nucleotides in length to specifically target mRNA (2) (Figure 1). It is known that 10-23 DNAzyme conducts catalytic cleavage on the inter-nucleotide phosphodiester bond between the unpaired purine $(\mathrm{A}, \mathrm{G})$ and the adjacent paired pyrimidine (U, C) (3). In comparison with antisense oligonucleotides and small interfering RNAs, Dzs show less off-target effects due to their high specificity and lack of immunogenicity in vivo
(4). As more results of clinical trials carried out so far are gradually presented, Dzs may turn out to be safe and well-tolerated therapeutics in human (5). Therefore, a deoxyribozyme drug may be available in the near future in the market (1).

Treatment of infections caused by drug-resistant strains of pathogenic bacteria is an important task for contemporary medicine. 10-23 Dzs were, therefore, proposed as potential antibacterial agents. Dzs were investigated against ampicillin-resistant E. coli strains with extended spectrum $\beta$-lactamase i.e. TEM-1 and TEM-3 (5) and Methicillin-resistant Staphylococcus aureus (MRSA) (6-8). Effective inhibition of ampicillin-resistant bacteria TEM1 and TEM3 has been demonstrated by novel mono-Dzs Dz1, Dz2, and bis-Dz Dz1-2 targeting $\beta$-lactamase mRNA (8). 
Dz1-2 showed the greatest inhibition effect, demonstrating that in vivo activity may be increased by constructing multiple-target Dzs.

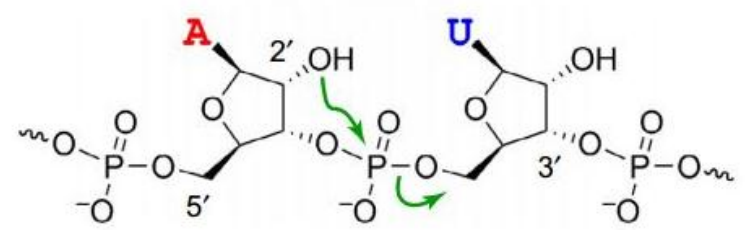

Figure 1. RNA cleavage reaction by 10-23 at AC junction.

The same strategy has been used by Hou and Unwalla in recent years $(9,10)$. A Dz with phosphorothioate linkages targeting blaR1 RNA was able to inhibit $\beta$-lactamase-mediated oxacillin resistance in Staphylococcus aureus through a significant decrease in transcription of blaR1 mRNA and marked reduction of blaZ. The results indicated that blocking the blaR1-blaZ signaling pathway by Dzs may provide a viable strategy for inhibiting $\mathrm{S}$. aureus resistance to $\beta$-lactam antibiotics and for combating MRSA (6). Restoration of antibiotic susceptibility in MRSA by targeting mecR1, a $\beta$ lactam-sensing transmembrane signaling protein, with phosphorothioate $\mathrm{Dz}$ was also demonstrated (7). The results of the study indicate that blocking the MecR1-MecI-MecA signaling pathway with amecR1-targeting Dz can restore the sensitivity of MRSA to existing $\beta$-lactam antibiotics. Dzs were designed to cleave Mycobacterium tuberculosis isocitrate lyase (ICL) mRNA in macrophages (11). Hidden infection of macrophages by $M$. tuberculosis is a considerable problem in tuberculosis therapy. The ICL protein plays an important role in latent $\mathrm{M}$. tuberculosis metabolism. It was shown that a Dz4 was able to suppress the expression of the ICL gene and decrease the viability of the bacteria in macrophages. One can conclude that Dzs may produce therapeutic agents for the treatment of bacterial infections. However, here, as well as for antivirals, many more studies are still required to be done to fully understand the biological effects of Dzs and to demonstrate their efficiency in vivo.

To design a $\mathrm{Dz}$ for a specific target, first the sequences of the target mRNAs are retrieved from available databases such as NCBI or addgene. The open reading frames (ORFs) of the retrieved sequences are then analyzed by ExPASy. The confirmed sequences of the target mRNA, either being the forward or reverse complement sequence, are then analyzed by DINAMelt web server, Mfold software which provides thermodynamic parameters on the folding of the single stranded nucleic acids and predicts the most stable secondary structures. The target sites are usually chosen inside loop regions or in positions that are not embedded inside extensive stem-loops.

The target mRNA in this study was chosen to be the $\alpha$-peptide that is used for $\alpha$-complementation and formation of blue color from lactose analogue X-gal (5-bromo-4-chloro-3-Ayndvlyl-beta-D-galactoside). Upon IPTG induction, the target mRNA is transcribed and consequently translated to $\alpha$-peptide to complement the activity of b-galactosidase. The active b-galactosidase hydrolyzes X-gal (12). X-gal hydrolysis leads to the production of galactose and 5bromo- 4-chloro-3-hydroxy-indole. The latter product dimerizes and is subsequently oxidized to a blue pigment (13). It is expected that the activity of Dz from this study i.e. cleavage of the target mRNA abolishes the translation of the $\alpha$-peptide and formation of the blue-color. In this respect, the bluewhite screening can be used to analyze the activity of Dz inside bacterial cells.

\section{Materials and methods}

Bioinformatics tools for definition of the target

The complete form of pGEM-T vector genetic map was taken from the addgene site. The lac $Z$ coding region is equivalent to $2832-2980$ regions in the addgene (www.addgene.org) in the reverse orientation. Coding region in mRNA of $\beta$-galactosidase was selected as a targeted sequence. The coding region ORFs were analyzed by ExPASy (www.expasy.org).

The secondary structure of mRNA of LacZ- $\alpha$ gene with the lowest free energy was taken from DINAMelt web server (unafold.rna.albany.edu) and Mfold software, RNA folding form (14).

Bioinformatics tools for modeling the designed $D z$ The designed Dzs were analyzed for non-specific binding to the host transcriptome by basic local alignment search tool (BLAST) from NCBI (www.ncbi.nlm.nih.gov/BLAST). The melting curve analysis of the designed Dz was performed by Mfold software.

\section{Results}

Selection of the target site for cleavage

According to the gene bank, the $\alpha$-peptide of the lacZ has the sequence with 126 amino acids as indicated in Table 1. The $\alpha$-peptide in the vectors that support $\alpha$ complementation consists of a fragment of such sequence. Here, we analyzed the sequence data of the pGEM-T vector. The selected vector, codes for the $\alpha$ peptide with a promoter which is in the reverse orientation. The related sequence of $\alpha$-peptide in this vector was 378 nucleotides long and is shown in Table 1. The transcribed mRNA consisted of ORFs 
from which only one was shown to be responsible for

the translation of the mRNA into the $\alpha$-peptide.

Table 1. Related sequences in this study.

\begin{tabular}{|c|c|c|c|}
\hline Name & Sequence & Sequence level & Accession number \\
\hline$\alpha$-peptide & $\begin{array}{l}\text { mtmitpsaqltltkgnkswsstavaaalelvdppgcrnsisslsipstsrggpvpnspysesyyarsl } \\
\text { avvlqrrdwenpgvtqlnrlaahppfaswrnseeartdrpsqqlrslngewtrpvaah }\end{array}$ & Amino acids & $\begin{array}{l}\text { Genebank } \\
\text { AAS77680.1 }\end{array}$ \\
\hline $\begin{array}{l}\text { Assigned sequence to } \\
\alpha \text {-peptide in addgene }\end{array}$ & $\begin{array}{l}\text { TCAGGCTGCGCAACTGTTGGGAAGGGCGATCGGTGCGGGCCT } \\
\text { CTTCGCTATTACGCCAGCTGGCGAAAGGGGGATGTGCTGCAA } \\
\text { GGCGATTAAGTTGGGTAACGCCAGGGTTTCCCAGTCACGAC } \\
\text { GTTGTAAAACGACGGCCAGTGAATTGTAATACGACTCACTAT } \\
\text { AGGGCGAATTGGGCCCGACGTCGCATGCTCCCGGCCGCCATG } \\
\text { GCCGCGGGATATCACTAGTGCGGCCGCCTGCAGGTCGACCAT } \\
\text { ATGGGAGAGCTCCCAACGCGTTGGATGCATAGCTTGAGTATT } \\
\text { CTATAGTGTCACCTAAATAGCTTGGCGTAATCATGGTCATAG } \\
\text { CTGTTTCCTGTGTGAAATTGTTATCCGCTCACAATTCCACACA } \\
\text { ACATACGAGCCGGAAGCATAAAGTGTAAA* }\end{array}$ & DNA & Addgene \\
\hline
\end{tabular}

The ORFs of this RNA are shown in Table 2. One of the three ORFs in the 3'-5' orientation had identity with position 67 to 115 of the $\alpha$-peptide of lacZ protein.
The predicted secondary structure of the transcribed mRNA had 16 loops that were interconnected by stems.

Table 2. Open reading frames of the transcribed RNA.

\begin{tabular}{|c|c|c|c|}
\hline Frame & Orientation & Sequence & Match with $\alpha$-peptide \\
\hline Frame 1 & $5^{\prime} 3^{\prime}$ & $\begin{array}{l}\text { S G C A T V G K G D R C G P LR Y Y A S W R K G D V L Q G D Stop V G Stop } \\
\text { R Q G F P H D V V KR R P V }\end{array}$ & no \\
\hline Frame 2 & $5^{\prime} 3^{\prime}$ & $\begin{array}{l}\text { Q A A Q L L GR A I G A G L F A I T P A G E R G Met C C K A I K L G N A R V } \\
\text { F P V T T L Stop N D G Q Stop }\end{array}$ & no \\
\hline Frame 3 & $5^{\prime} 3^{\prime}$ & $\begin{array}{l}\text { RLRNCWEGRSVRASS LLR QLAKGGCAARRLSWVTPGF } \\
\text { SQSRRCKTTASE }\end{array}$ & no \\
\hline Frame 1 & $3^{\prime} 5^{\prime}$ & $\begin{array}{l}\text { F T G R R F T T S Stop L G K P W R Y P T Stop S P C S T S P F R Q L A Stop } \\
\text { Stop R R G P H R S P F P T V A Q P }\end{array}$ & no \\
\hline Frame 2 & $3^{\prime} 5^{\prime}$ & 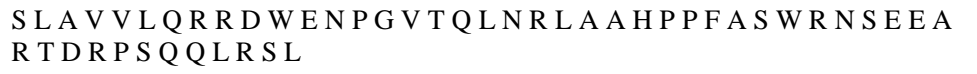 & Yes \\
\hline Frame 3 & $3^{\prime} 5^{\prime}$ & $\begin{array}{l}\text { H W P F Y N V V T G K T L A L P N L I A LQ H I P L S P A G V I A KR P A P } \\
\text { I A L P S C A A Stop }\end{array}$ & no \\
\hline
\end{tabular}

The predicted structure is shown in Figure 2A and its related energy plot is shown in Figure 2B. In the energy plot, in the upper triangular region, a dot in row $\mathrm{i}$ and column $\mathrm{j}$ represents a base pair between the $\mathrm{i}^{\text {th }}$ and $\mathrm{j}^{\text {th }}$ bases. The dots represent the superposition of all possible foldings. The mRNA had a loop starting from 56th nucleotide of the coding region. The A62C63 in this region was an appropriate candidate for cleavage since no extensive stem was present at this region. The selected cleavage site in the RNA of the lacZ gene sequence is defined in Figure 3A

\section{Designing $D z$}

For designing the Dz, either of binding arms have been designed to be 9 nucleotides long. The length of the binding arms has been chosen to be 9 based on a previous study. The overall catalytic efficiency of each deoxyribozyme as measured by kcat/KM values displayed a significant amount of variability between the modified and unmodified species. In the short arm deoxyribozymes ( $7-7 \mathrm{bp}$ ) the inclusion of an inverted base modification produced a 3 -fold decrease in the in vitro characterization of deoxyribozymes. In contrast to this negative effect on the cleavage activity, the relative efficiency of the long (9 - 9 bp) arm version was enhanced 10 -fold by the presence of inverted base modification. The intermediate length ( 8 - $8 \mathrm{bp}$ ) binding arm deoxyribozyme was the least affected by modification, showing a 2 -fold increase in the value of $\mathrm{kcat} / \mathrm{KM}$ (15). There should not be similar sequences in host bacteria. This was checked using the server NCBI. Similar sequences in the host genome cause the loss of bacteria, probably if it is in critical host genes. This also can affect the survival of the bacteria. Finally, deoxyribozyme binding was predicted by the Mfold server. Tm is an important parameter in the deoxyribozyme design. 


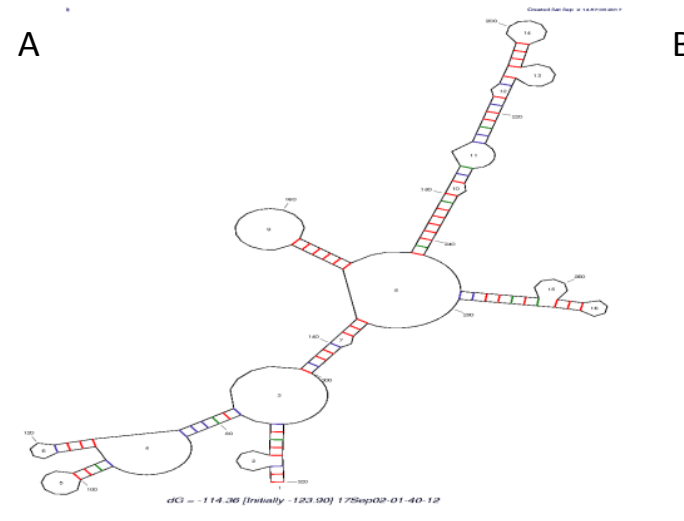

B

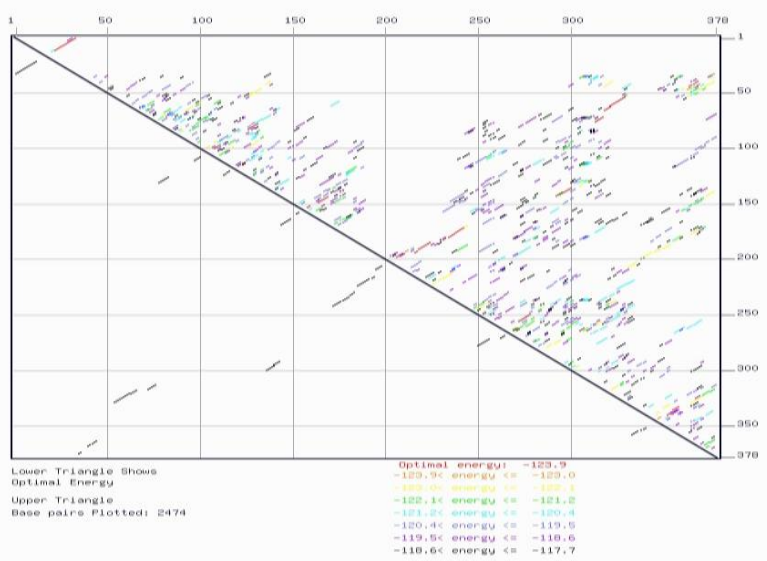

Figure 2. (A) Predicted secondary structure of the target mRNA. (B) Energy plot of the predicted structure.

This parameter indicates the temperature at which $50 \%$ oligonucleotides are attached to the model. Tm A

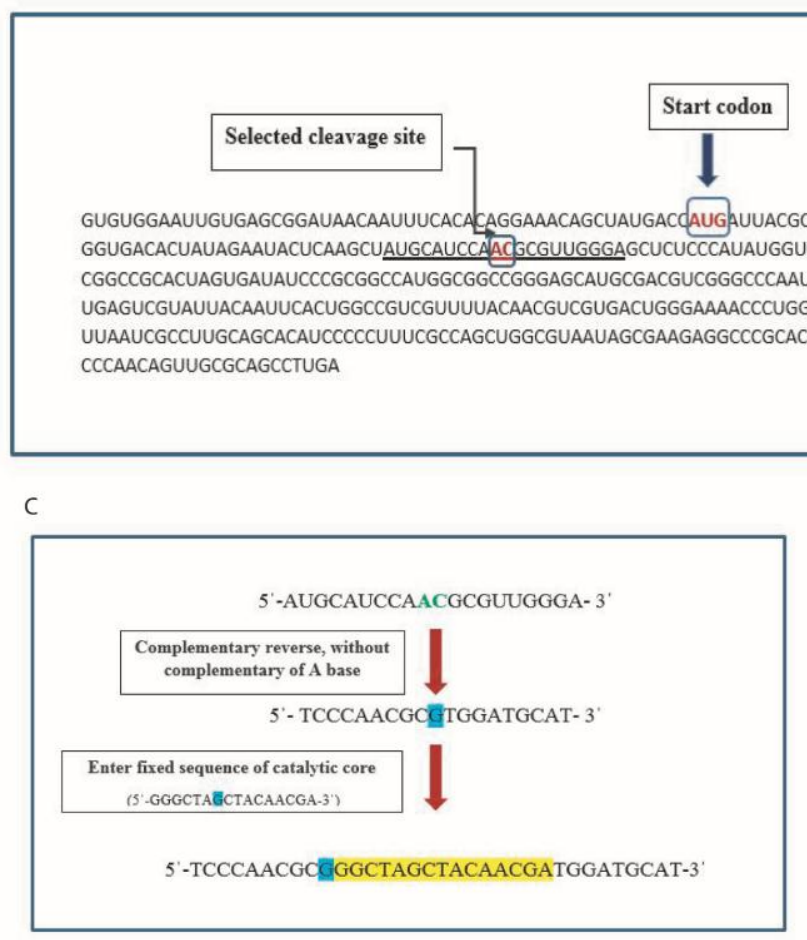

depends on the oligonucleotide length and its sequences.
B

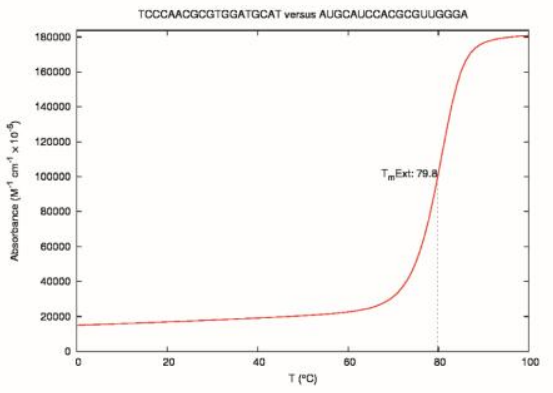

Figure 3. (A) Selected cleavage site in $L a c Z$ gene RNA sequence. (B) Measurement of designing 10-23 deoxyribozyme Tm (Mfold). (C) Determining deoxyribozyme sequence (D) final structure designing of 10-23 deoxyribozyme.

The higher the GC content of the two arms, the lower is the free energy of the hybridization of the DNAzyme-substrate heteroduplex. Therefore, the binding stability of the heteroduplex will be higher. There are several formulas to obtain the Tm. Different databases for $\mathrm{Tm}$ calculators can be used to assess specific oligonucleotide $\mathrm{Tm}$. The Tm temperature resulting from deoxyribozyme binding to the target

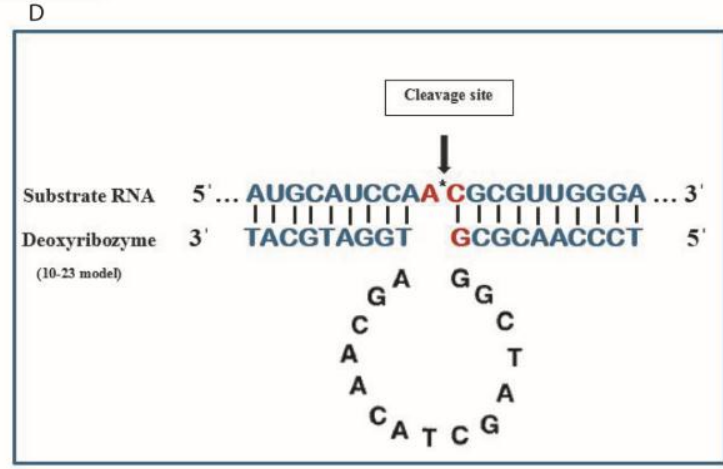

site was measured as 79.8 by Mfold (Figure 3B). Tm of binding arms to their target was also calculated separately. The result showed 60.6 and 47.3 for Tm of the left arm and right arm respectively. It would determine whether after the reaction and mRNA cleavage, two pieces of target separated from binding arms or not. In the next step, the target site sequence was reversed complementary. At this step, 10-23 
deoxyribozyme is cut between a paired purine and an unpaired pyrimidine. So the complement of A base doesn't place at $\mathrm{AC}$ cleavage site in the enzyme sequence to keep A base as unpaired. And then the 10-23 deoxyribozyme catalytic core which has a fixed sequence was added in the middle of the sequence. This process is shown in Figure $3 \mathrm{C}$ and the final structure designing of 10-23 deoxyribozyme is shown in Figure 3D, respectively.

\section{Discussion and conclusion}

Recently for inhibition of gene expression at the mRNA level, a variety of reagents including antisense oligonucleotides, small interfering RNA(16), branched RNAs (17), ribozyme (18) and deoxyribozyme have been investigated. Among all of these, DNA due to its properties has been considered as a catalyst more than RNAs and proteins. Evidence has shown primary requisite, performance and potential of Dzs in different diseases including diseases associated with viruses, bacteria and cancer (1). Treating infections caused by pathogen drug-resistant strains is one of the most important tasks in the new generation of drugs. 10-23 $\mathrm{Dz}$ is assumed as an appropriate antibacterial reagent (1). Breaking target mRNA involved in drug resistance with the help of the DNAzymes is a convenient way to sensitize bacteria to drug-resistance against antibiotics. The mRNA of $\beta$-galactosidase is one of the most important objectives that has been selected in this study, due to the fact that $\beta$ galactosidase is responsible for producing resistance to beta-galactosidase antibiotics.

In this study, the properties of the lactose operon were used to evaluate the performance of 10-23 deoxyribozyme within prokaryotic cells perhaps deoxyribozyme was designed against coding region of lacZ. Researches showed that the deoxyribozyme modified by a $3^{\prime}-3^{\prime}$ inversion at the $3^{\prime}$ end had substantially greater stability compared with the unmodified deoxyribozyme and the effective concentration range of the most active molecule (9/9 arms with 3'- inverted base modification) is more effective than other arms with different lengths and could cause significant suppression (15).

According to the results of this study, the ability of 10-23 deoxyribozyme in mRNA of LacZ- $\alpha$ genes encoding region site in bacterial cells can be examined. In vitro and in vivo investigation of the functionality of 10-23 deoxyribozyme is under investigation by our group.

\section{Author Contributions}

AE, FJZ conceived of the presented idea. NA performed the computations. AE, FJZ verified the analytical methods. All authors discussed the results and contributed to the final manuscript.

\section{Acknowledgements}

This work was supported by the grant from the University of Isfahan.

\section{Conflict of Interest}

No conflict of interest exists.

\section{References}

1. Fokina AA, Stetsenko DA, François J-C. DNA enzymes as potential therapeutics: towards clinical application of 10-23 DNAzymes. Expert Opin Biol Ther. 2015; 15, 689-711. PMID: 25772532

2. Wang $X$, Zhang L, Ding N, Yang X, Zhang J, He J, et al. Identification and characterization of DNAzymes targeting DNA methyltransferase I for suppressing bladder cancer proliferation. Biochem Biophys Res Commun. 2015; 461(2):329-33. PMID: 25888794

3. Zhu J, Li Z, Yang Z, He J. Studies on the preferred uraciladenine base pair at the cleavage site of 10-23 DNAzyme by functional group modifications on adenine. Bioorg Med Chem. 2015; 23(15):4256-63. PMID: 26145822

4. Fokina AA, Stetsenko DA, Francois JC. DNA enzymes as potential therapeutics: towards clinical application of 10-23 DNAzymes. Expert Opin Biol Ther. 2015; 15(5):689-711. PMID: 25772532

5. Krug N, Hohlfeld JM, Kirsten A-M, Kornmann O, Beeh KM, Kappeler D, et al. Allergen-Induced Asthmatic Responses Modified by a GATA3-Specific DNAzyme. N Engl J Med. 2015; 372(21):1987-95. PMID: 25981191

6. Hou Z, Meng JR, Zhao JR, Hu BQ, Liu J, Yan XJ, et al. Inhibition of beta-lactamase-mediated oxacillin resistance in Staphylococcus aureus by a deoxyribozyme. Acta Pharmacol Sin. 2007; 28(11):1775-182. PMID: 17959028

7. Hou Z, Meng JR, Niu C, Wang HF, Liu J, Hu BQ, et al. Restoration of antibiotic susceptibility in methicillin-resistant staphylococcus aureus by targeting mecr1 with a phosphorothioate deoxyribozyme. Clin Exp Pharmacol Physiol. 2007; 34(11):11604. PMID: 17880371

8. Chen F, Li Z, Wang R, Liu B, Zeng Z, Zhang H, et al. Inhibition of ampicillin-resistant bacteria by novel mono-DNAzymes and diDNAzyme targeted to $\beta$-lactamase mRNA. Oligonucleotides. 2004; 14(2):80-9. PMID: 15294072

9. Hou W, Ni Q, Wo J, Li M, Liu K, Chen L, et al. Inhibition of hepatitis $\mathrm{B}$ virus $\mathrm{X}$ gene expression by 10-23 DNAzymes. Antiviral Res. 2006; 72(3):190-6. PMID: 16930733

10. Unwalla H, Banerjea AC. Novel mono-and di-DNA-enzymes targeted to cleave TAT or TAT-REV RNA inhibit HIV-1 gene expression. Antiviral Res. 2001; 51(2):127-39. PMID: 11431037

11. Li J, Zhu D, Yi Z, He Y, Chun Y, Liu Y, et al. DNAzymes targeting the icl gene inhibit ICL expression and decrease Mycobacterium tuberculosis survival in macrophages. Oligonucleotides. 2005; 15(3):215-22. PMID: 16201909

12. Horwitz JP, Chua J, Curby RJ, Tomson AJ, Da Rooge MA, Fisher BE, et al. Substrates for Cytochemical Demonstration of 
Enzyme Activity. I. Some Substituted 3-Indolyl- $\beta$-Dglycopyranosides1a. J Med Chem. 1964; 7(4):574-5.

13. Langley KE, Villarejo MR, Fowler AV, Zamenhof PJ, Zabin I. Molecular basis of beta-galactosidase alpha-complementation. Proc Natl Acad Sci U S A. 1975; 72(4):1254-7. PMID: 1093175

14. Markham NR, Zuker M. DINAMelt web server for nucleic acid melting prediction. Nucleic Acids Res. 2005; 33(Web Server issue):W577-81. PMID: 15980540

15. Sun L-Q, Cairns MJ, Gerlach WL, Witherington C, Wang L, King A. Suppression of smooth muscle cell proliferation by a c- myc RNA-cleaving deoxyribozyme. J Biol Chem. 1999; 274(24):17236-41. PMID: 10358082

16. Abe H. [Nanostructured RNA for RNA interference] Yakugaku Zasshi. 2013; 133(3):373-8. PMID: 23449417

17. Nakashima Y, Abe H, Abe N, Aikawa K, Ito Y. Branched RNA nanostructures for RNA interference. Chem Commun (Camb). 2011; 47(29):8367-9. PMID: 21691656

18. Burnett JC, Rossi JJ. RNA-based therapeutics: current progress and future prospects. Chem Biol. 2012; 19(1):60-71. PMID: 22284355 